\title{
Mesh independency study for an elementary perforated panel part of an air solar collector
}

\author{
Andrei-Stelian Bejan ${ }^{1,2^{*}}$, Florin Bode ${ }^{2,3}$, Tiberiu Catalina ${ }^{1,2}$ and Cătalin Teodosiu ${ }^{1}$ \\ ${ }^{1}$ Technical University of Civil Engineering Bucharest, Faculty of Engineering for Building Services, 021414 Bucharest, Romania \\ ${ }^{2}$ CAMBI Research Center, 021407 Bucharest, Romania \\ ${ }^{3}$ Technical University of Cluj-Napoca, 400114 Cluj-Napoca, Romania
}

\begin{abstract}
In order to achieve the numerical model of a transpired solar collector (TSC) with integrated phase changing materials (PCM) it is mandatory to study the impact of the orifice geometry on the entire system. The numerical simulation of the entire solar collector absorber metal plate $(1000 \times 2000 \mathrm{~mm}$ and 5000 orifices) is not feasible thus resulting a huge number of cells for the numerical grid for which we will need very high computational resources and a very large amount of time to be solved. By taking these aspects into account we decided to simulate only four equivalent orifices and then to transpose the results to the actual case for further studies. The present paper aims to analyse the mesh independency study for an elementary perforated panel with four equivalent lobed orifices which is part of a real case TSC. This analysis represents one of the most important stages within the construction of the TSC numerical model and doesn't need an experimental validation. The study was conducted in Ansys Fluent CFD software and the results were processed directly by using Tecplot software. Six different meshes were analysed (from 0.2 to 7.3 million cells), boundary conditions were imposed, and $\mathrm{k}-\varepsilon$ RNG turbulence model was used according to the literature. After comparing velocity and temperature fields in longitudinal and transverse planes we concluded that from 5.3 million cells the solution is independent of the meshing quality.
\end{abstract}

\section{Introduction}

\subsection{Objective}

Regardless of their type, the operating principle of air solar collectors is the same [1]. Solar radiation is captured by an absorbent element (generally a metal plate) that transfers the heat to the airflow within the collectors' air cavity.

In the case of an opaque solar collectors, the heat is usually captured by a planar metal panel (absorber) or a black perforated panel, then it is released to the air which is then collected at the top of the solar collector and introduced into the room by a fan (in winter or during transition periods). If there are inertial elements, the energy stored during the daytime can be later released at night. During the daytime, in summer, the solar collector can work as a thermal buffer that reduces the cooling loads determine by the building envelope or as a ventilated facade with the same role.

Transpired solar collectors (TSCs) typically have a lower investment costs and high efficiency in the case of heating systems, fresh air preheating systems or drying systems [2] and moreover, they have lower lifetime costs [3]. Opaque solar collectors are typically used for applications where the required temperature is lower [4] and side winds are not very strong [5].
A numerical study conducted by Van Decker et al. [6] on a TSC shows that about $62 \%$ of the thermal energy is transferred to the air in front of the absorbent plate, $28 \%$ in the orifices (so the geometry of orifices has an important role) and about $10 \%$ behind the plate. The thermal transfer efficiency decreases as the air velocity increases and the orifices pitch increases. Moreover, the thermal transfer efficiency increases as the wind speed increases and if the absorber plate is thicker.

In order to optimize the geometry and parameters of a TSC it is mandatory to run numerical simulations. According to the literature one of the software often used to simulate the air solar collectors is Ansys Fluent CFD software [7-10].

The present study is part of a complex numerical study regarding the implementation of phase changing materials in transpired air solar collectors with lobed orifices which are acting as a solar wall.

As we can see above the geometry of the orifice is very important and its impact cannot be neglected. A lot of researches regarding the flow through different orifices have been carried out [11-14]. We used lobed shaped orifices for the study taken in this article, because according to the literature $[1,15]$, the lobed geometries (unlike the classical circular ones) are $15 \%$ more efficient for flows ranging from $80 \mathrm{~m}^{3} / \mathrm{h} / \mathrm{m}^{2}$ to $220 \mathrm{~m}^{3} / \mathrm{h} / \mathrm{m}^{2}$ and can determine an outlet air temperature higher with up to $2^{\circ} \mathrm{C}$. The unconventional shape of the

\footnotetext{
* Corresponding author: andrei.s.bejan $@$ gmail.com
} 
lobed orifices determines a more complex flow that improves heat transfer by approximately $40 \%$. The geometry of the entire solar collector is very complex and it is presented in the following chapters. By simulating the entire solar collector absorber, we obtained a large number of cells which determine a large period of time needed in order to compute. Moreover, the computational resources must be much higher. By taking these aspects into account we decided to simulate only four equivalent orifices and then to transpose the results to the real case for further studies.

The current paper main objective is to analyse the mesh independency study for an elementary perforated panel with four equivalent lobed orifices which is part of a real case TSC.

\subsection{Methodology}

Computational Fluid Dynamics (CFDs) is a widely used numerical modelling technique in the engineering and research. CFDs technique is based on the fundamental equations governing fluid dynamics: the continuity equation, the energy conservation equation and the impulse conservation equation. Because the results of Navier-Stokes equations cannot be solved analytically, the CFD software tries to find an approximate solution by spatial meshing methods that convert partialderivative equations into algebraic equations (calculations are iterative).

As mentioned before, ANSYS Fluent [16] is one of the most used CFD software and it is based on the finite volume method which splits the entire volume in small volumes thus creating a spatial mesh.

The steps of a CFD numerical simulation that were followed in the present paper are:

a)Creating the geometry of the model

b)Creating the calculation grid - mesh independency study

c)Imposing boundary conditions and configuring the numerical case

d) Validating the numerical model by comparing the numerical results with the experimental ones

e) Calculation of the solution and post-processing.

The present paper cover the first three steps and the following two steps regarding the model validation and post-processing will be analysed in further studies which will be conducted in the future.

Numerical simulation software CFD ANSYS Fluent and all its components: ANSYS DesignModeler, ANSYS Workbench and ANSYS Mesher were used to carry out the numerical study and Tecplot 360; Notepad and Microsoft Excel were used for data processing. Moreover, one computing station which is in the endowment of Faculty of Building Service Engineering laboratories was used (Intel Xeon 3.4Ghz, 16 cores, 128 GB RAM).

\section{Material and method}

\subsection{Geometry}

In order to be able to perform the numerical modelling of the solar collector with lobed perforations and integrated PCMs in a feasible period of time, we have simplified as much as possible the geometry of the absorber plate that is part of the solar collector, starting from the premise of the thermal transfer phenomena symmetry and velocity fields symmetry.

As it could be observed from the literature, the shape of the orifices considerably influences the efficiency of the solar collector [1, 15], as it can improve thermal transfer and can cause a higher air temperature in the collector cavity. The unconventional lobed geometry of the absorbent plate orifices determines complex airflows thus improving the heat transfer and according to the literature is the geometry that helps to obtain the greatest rise in temperature between the ambient air (inlet) and the exhaust air (outlet).

Taking into account all these arguments, we decided to consider the effect of orifices on the solar collector flow and efficiency. Moreover, this approach will create future premise of studies regarding the orifices influence on the PCMs integration.

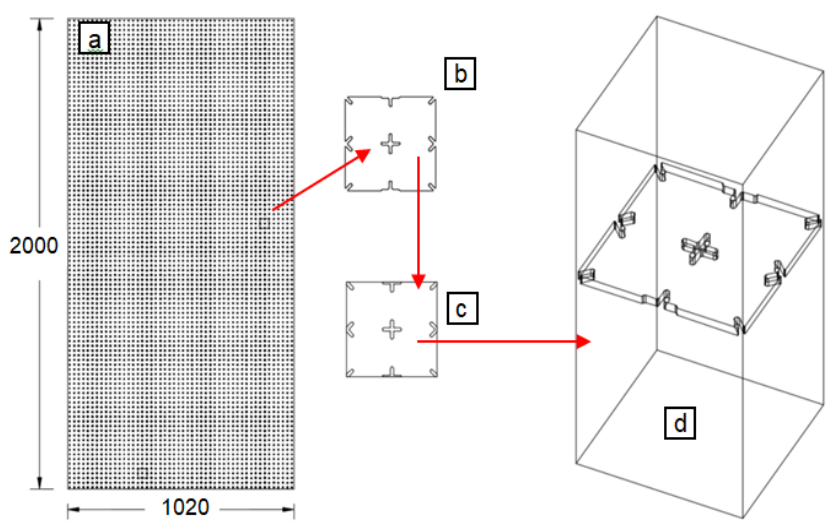

Fig. 1. Designing the 3D model in AutoCAD: $a-a b s o r b e n t$ metal plate with lobed orifices, $b$ - extraction of a $4 \times 4 \mathrm{~cm}$ part, $\mathrm{c}-$-embedment in the computing domain, $\mathrm{d}-3 \mathrm{D}$ computing domain $(4 \mathrm{x} 4 \mathrm{~cm}$ plate surrounded by the computational domain).

Figure 1 shows the layout and geometry of the lobed holes. The orifices are alternately positioned horizontally $(+, \mathrm{x},+, \mathrm{x},+\ldots$ etc. $)$ at a pitch of $20 \mathrm{~mm}$ and with an equivalent diameter of $5 \mathrm{~mm}$.

As it can be observed in Figure 1a, the absorbent plate with a surface area of approximately $2 \mathrm{~m}^{2}$ and a thickness of $2 \mathrm{~mm}$ has a large number of lobed orifices on its entire area (5000 holes). Modelling the entire absorbent plate with lobed holes would require a complex mesh and a large number of mesh cells in the computing domain in order to accurately capture the phenomena that occur near the orifices. This would lead to important CPU time and very high computational resources necessary in order to carry out the numerical studies.

Considering the airflow symmetry and the transfer phenomena symmetry we selected a $4 \times 4 \mathrm{~cm}$ metal plate containing 4 equivalent orifices for the present study (Figure 1b). 
The $4 \times 4 \mathrm{~cm}$ extracted metal plate was then enclosed in a $9.2 \times 4 \times 4 \mathrm{~cm}$ parallelepiped that will represent the airflow in the computing domain, with respect to the flow direction (Figure 1c and 1d).

The plate is positioned at $4 \mathrm{~cm}$ distance from the computing domain limit (inlet - upstream) and at $5 \mathrm{~cm}$ distance from the outlet (downstream) which represents ten equivalent diameters $(10 \mathrm{De}=5 \mathrm{~cm})$.

From the experimental studies carried out at the Faculty of Building Services Engineering we noticed that after $10 \mathrm{De}$, in the case of lobed orifices the airflow and velocity profiles are stabilizing. The velocity fields thus obtained at a distance of $5 \mathrm{~cm}$ from the metal plate will then be used (introduced as boundary limits on the inlet) into the simplified numerical model of the solar collector.

The 3D geometry was designed in AutoCAD and converted in .igs format that can be imported in ANSYS DesignModeler (Figure 2).
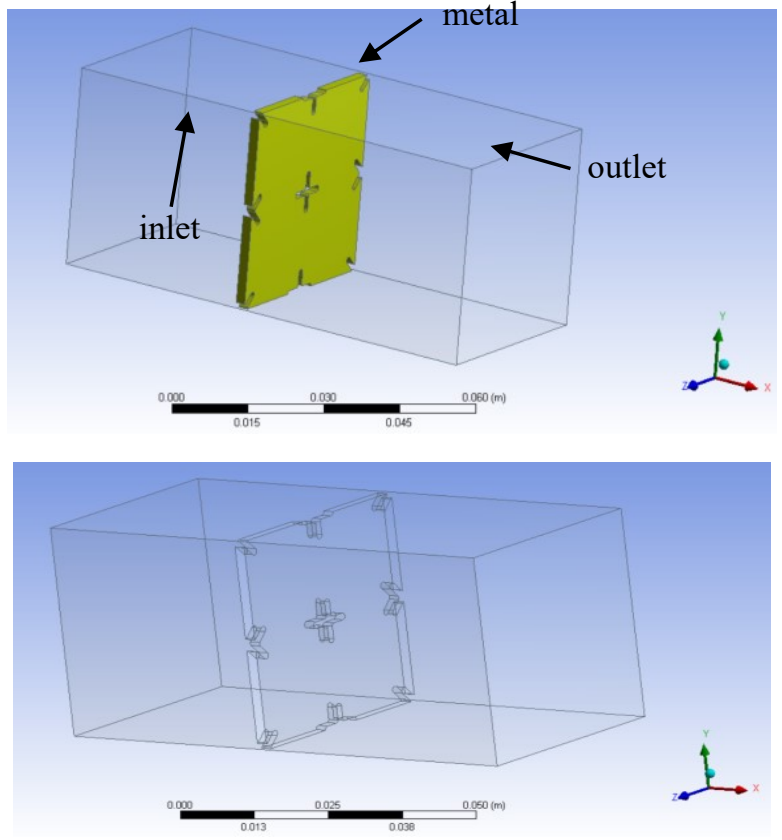

Fig. 2. Importing the geometry from AutoCAD to ANSYS DesignModeler - isometric view.

Taking into account that the $2 \mathrm{~mm}$ thick metal plate is made of aluminium which has high thermal conductivity, the heat transfer is very fast thus resulting a relatively uniform temperature across the surface.

The internal transfer phenomena are not very complex and do not influence the result of the simulation. Therefore, the $4 \times 4 \mathrm{~cm}$ metal plate was extracted from the computing domain, taking into account only the heat transfer due to the air flow around the aluminium plate.

Once the geometry has been built as explained above, it was imported into ANSYS Meshing in order to achieve spatial meshing.

\subsection{Meshing}

After building the geometry, the next step in creating the numerical model is the choice of the optimum mesh.
In order to determine the number of computing elements (cells) useful to solve the problem, it is necessary to make a solution independency study based on the quality of the mesh or mesh independency study.
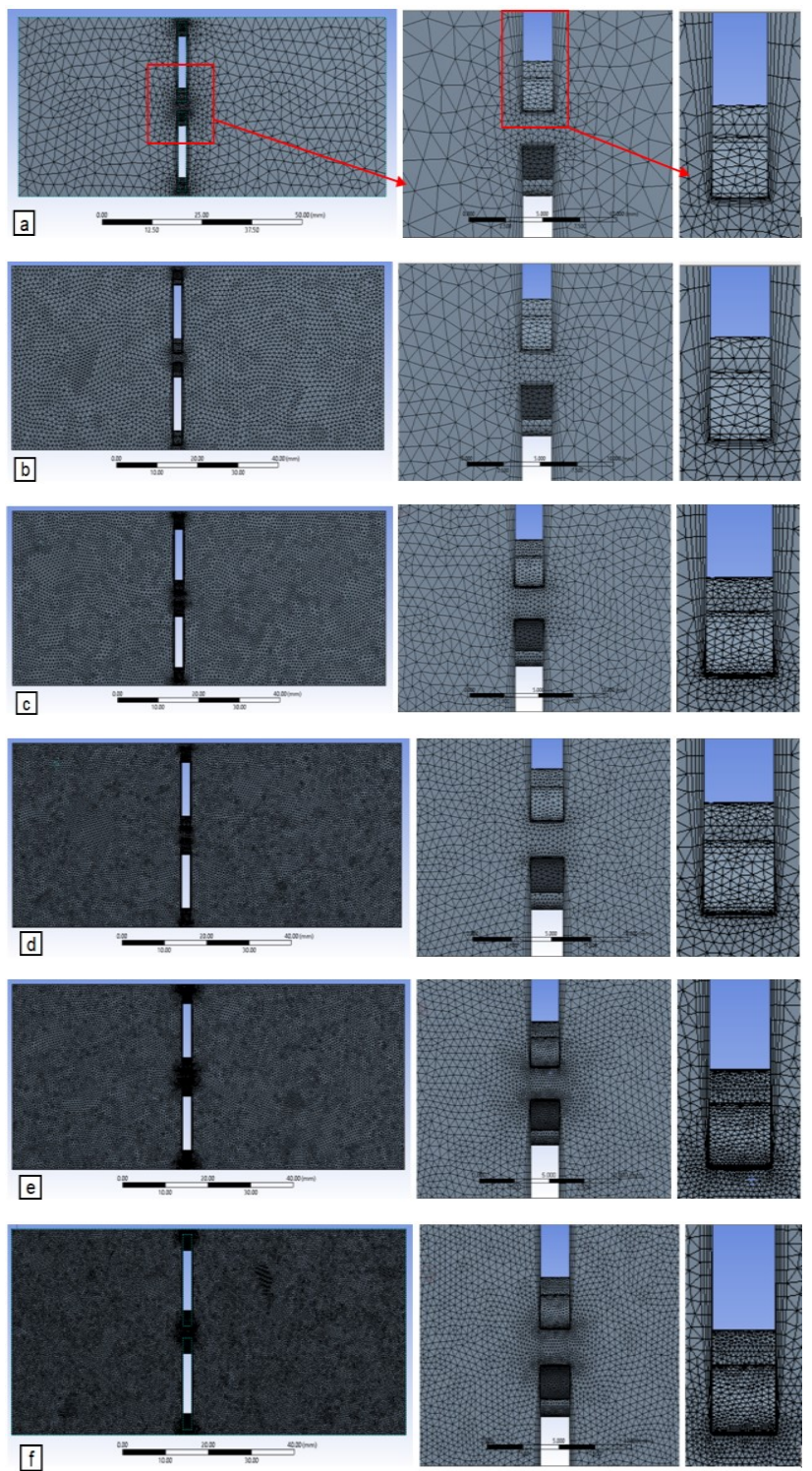

Fig. 3. Different mesh levels for six different cases analysed: overview, orifice detail and boundary detail for a) 0.2 , b) 0.62 , c) 1.5, d) 3 , e) 5.3, f) 7.3 million elements.

The study aims to achieve a correct numerical result, but the accuracy of the results is directly related to the number of cells (more cells $=$ more accurate result). Furthermore, if the number of cells is higher, the calculation time becomes higher and the necessary computing resources will also increase.

The mesh independency study begins by choosing multiple levels of meshing with more and more cells. From a certain number of cells up, the results obtained vary insignificantly, this computational grid representing the optimal solution regarding the result-computing ratio.

In order to conduct the mesh independency study we have chosen 6 levels of meshing (Figure 3), respectively: $0.2,0.62,1.5,3,5.3$ and 7.3 million tetrahedral 
computing elements. The optimum meshing level will be chosen by conducting individual numerical simulations, using similar boundary conditions and cases.

\subsection{Boundary conditions and the case setup}

The six meshing levels were sequentially imported into ANSYS Fluent and for each we imposed the same boundary conditions as presented in Table 1 .

The air velocity (in the inlet area) is $0.05555 \mathrm{~m} / \mathrm{s}$ corresponding to the nominal airflow rate of $400 \mathrm{~m} 3 / \mathrm{h}$ for the entire collector (according to the equations below), the inlet air temperature is $25^{\circ} \mathrm{C}$ and the metal plate temperature is $5^{\circ} \mathrm{C}$ (corresponding to a solar radiation value of approximately $530 \mathrm{~W} / \mathrm{m}^{2}$ according to the experimental studies conducted).

Table 1. Boundary conditions used for the numerical case.

\begin{tabular}{|c|c|c|}
\hline Boundary condition & Value & Unit \\
\hline $\begin{array}{c}\text { Average velocity at the computing } \\
\text { domain limit (velocity inlet) }\end{array}$ & 0.05555 & $\mathrm{~m} / \mathrm{s}$ \\
\hline Inlet temperature (inlet) & 25 & ${ }^{\circ} \mathrm{C}$ \\
\hline Metal plate temperature (metal) & 50 & ${ }^{\circ} \mathrm{C}$ \\
\hline
\end{tabular}

After activating the energy model option, for the turbulence model we chose the k- $\varepsilon$ RNG model (with Enhanced Wall Function - EWF) which is suitable, according to the literature studied [17], in the cases with swirling flows and in the case of flows with small Reynolds numbers $(\operatorname{Re} \approx 422$ in the present study according to the equations below - laminar flow).

Having the temperature values at different points from the experimental study, we did not use the heat transfer model for radiation in order to obtain a simplified model and to facilitate as much as possible the calculations. This model will be developed in the further studies and will underpin the continuation of postdoctoral studies.

With respect to the above presented arguments, we performed several numerical simulations for the 6 computational grids chosen, the results being imported into Tecplot software and analysed further.
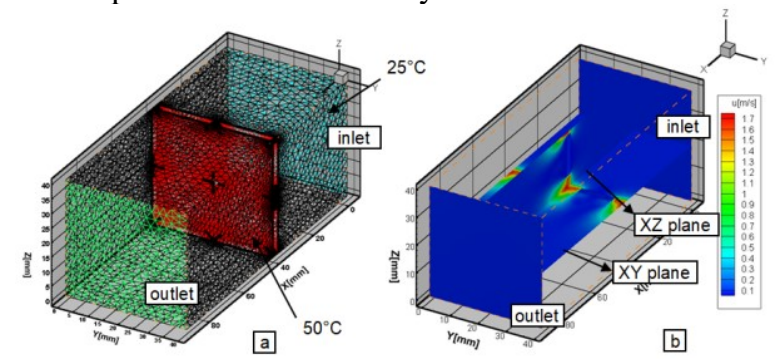

Fig. 4. Importing results in Tecplot 360 in order to be properly processed.

Figure 4 presents the results imported into the Tecplot data processing software. Figure 4a shows the cell distribution for one of the six cases and Figure $4 \mathrm{~b}$ shows the velocity magnitude distribution in two longitudinal planes: $\mathrm{XY}$ and $\mathrm{XZ}$. Further, the longitudinal and transvers velocity fields will be analysed for each of the 6 cases mentioned above.

\section{Results and discussions}

\subsection{Velocity and temperature fields - longitudinal $X Y$ planes}

After conducting various numerical simulations with Ansys Fluent using the conditions and settings mentioned above we have obtained the results emphasised in the following figures.

Figure 5 presents the velocity fields and figure 6 emphasise the temperature fields for the XY longitudinal plane.

As it can be observed in Figure 5, the velocity profiles on XY longitudinal planes are relatively similar for all 6 cases without important differences. Moreover, as it can be noticed from figure 6, the temperature profiles in the XY longitudinal planes are similar in all the 6 cases and we cannot precisely determine the optimal solution for the spatial meshing.

By taking into account these results, we will further analyse the velocity fields at 5De distance from the metal plate and 10De distance from the metal plate in transverse planes.

\subsection{Velocity fields - 5De and 10De transverse planes}

Figure 7 shows the velocity fields at 5De distance from the metal plate for all six analysed cases. It can be noticed that starting with 3 million elements, there are no significant variations regarding the velocity fields.
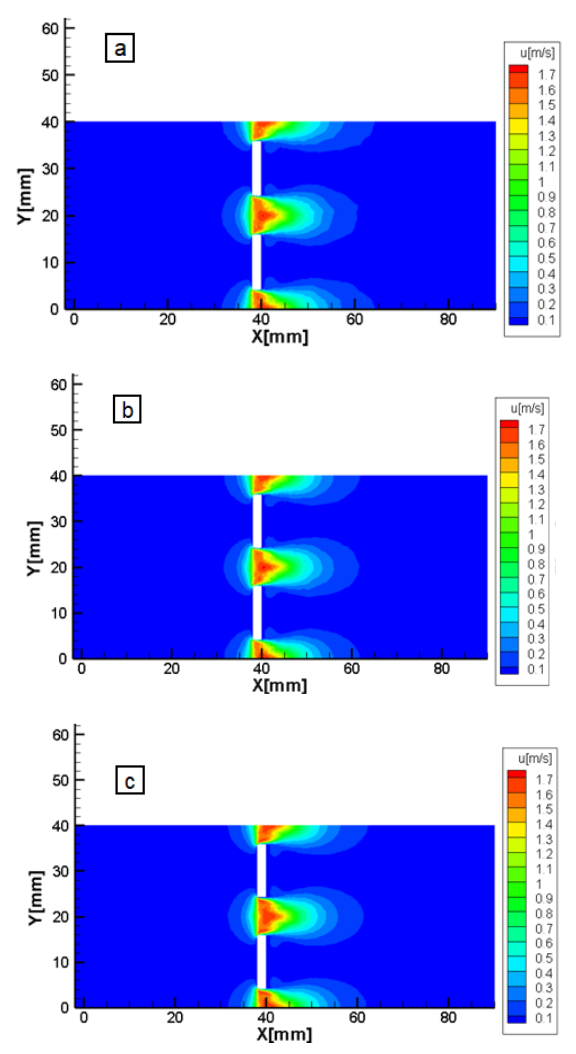

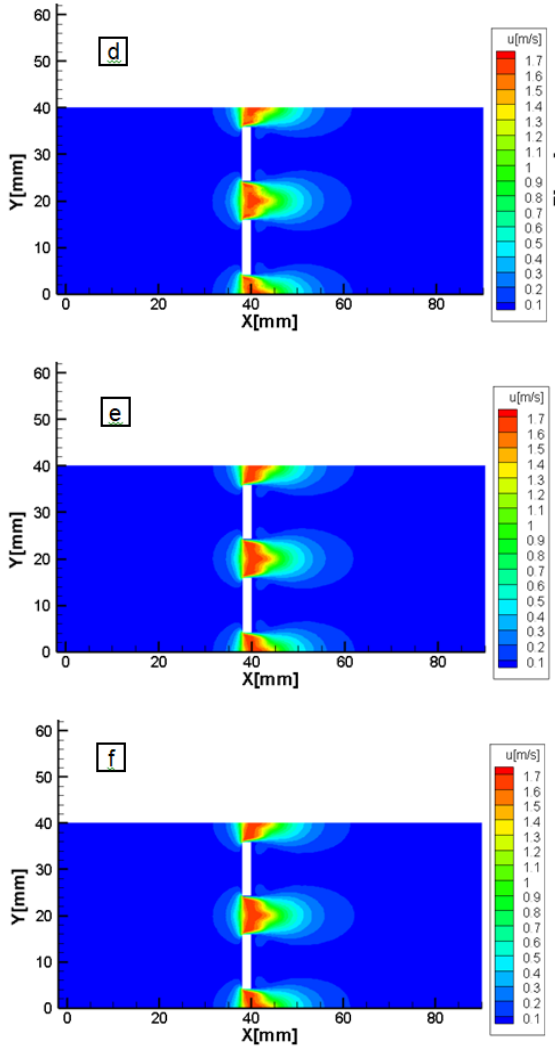

Fig. 5. (continuation) Velocity fields $(\mathrm{u}[\mathrm{m} / \mathrm{s}]$ - velocity magnitude) for $\mathrm{XY}$ longitudinal plane for all the six cases a) 0.2 , b) 0.62 , c) 1.5 , d) 3 , e) 5.3 , f) 7.3 million cells.
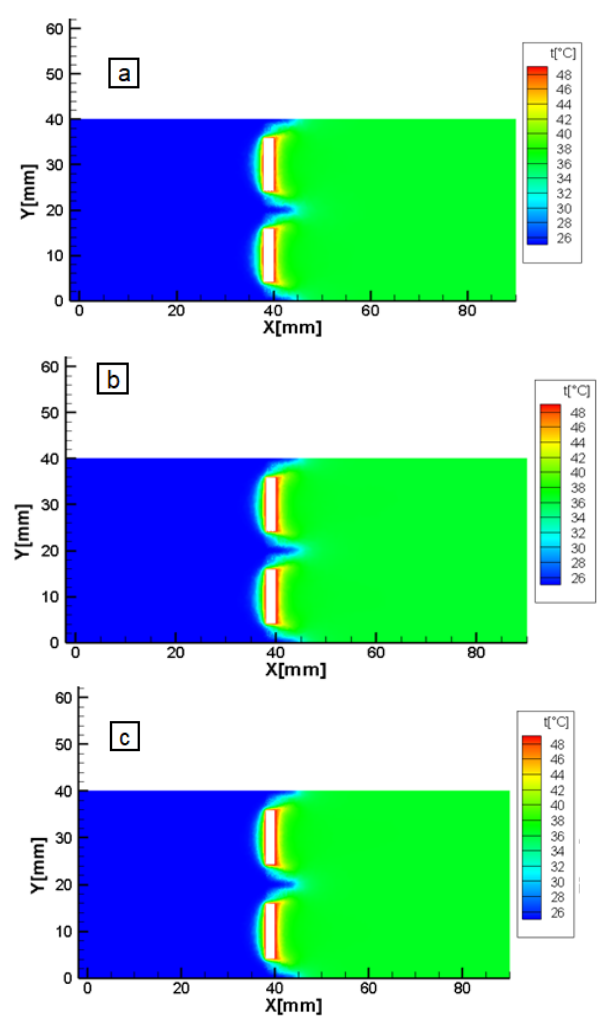
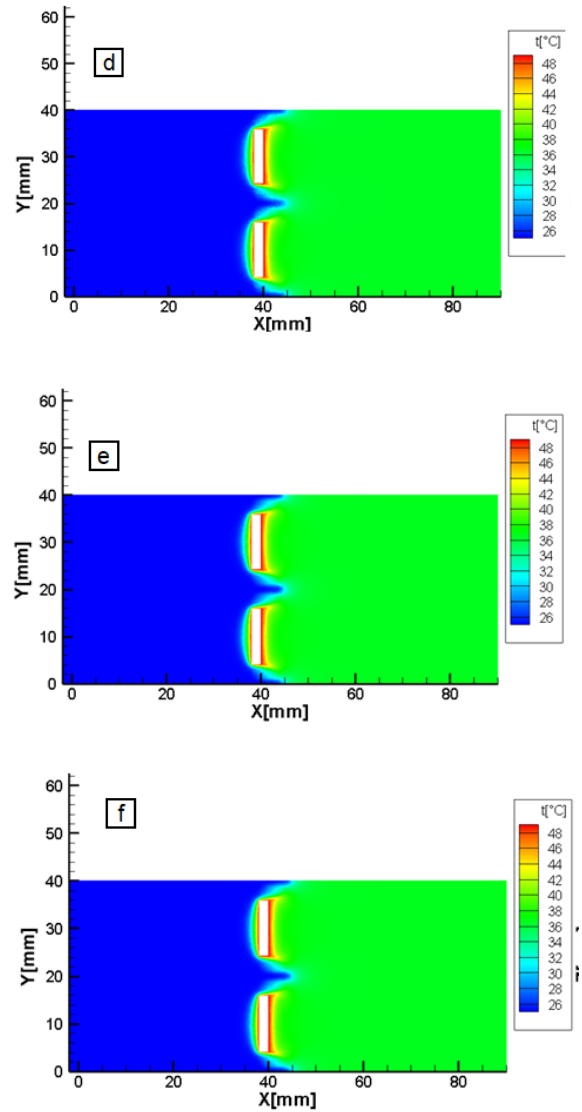

Fig. 6. Temperature field for XY longitudinal plane for all the six cases a) 0.2 , b) 0.62 , c) 1.5 , d) 3 , e) 5.3 , f) 7.3 million cells

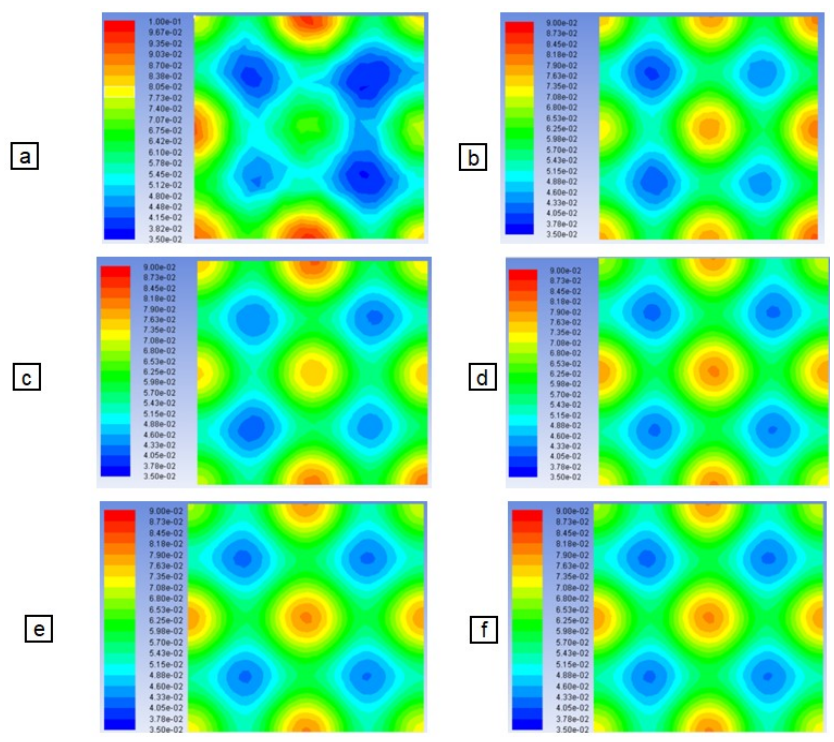

Fig. 7. Velocity fields $(\mathrm{u}[\mathrm{m} / \mathrm{s}]$ - velocity magnitude) in transverse plane at distance $5 \mathrm{De}(2.5 \mathrm{~cm})$ from the metal plate in all the six cases analysed: a) 0.2 , b) 0.62 , c) 1.5 , d) 3 , e) 5.3 , f) 7.3 million elements. 


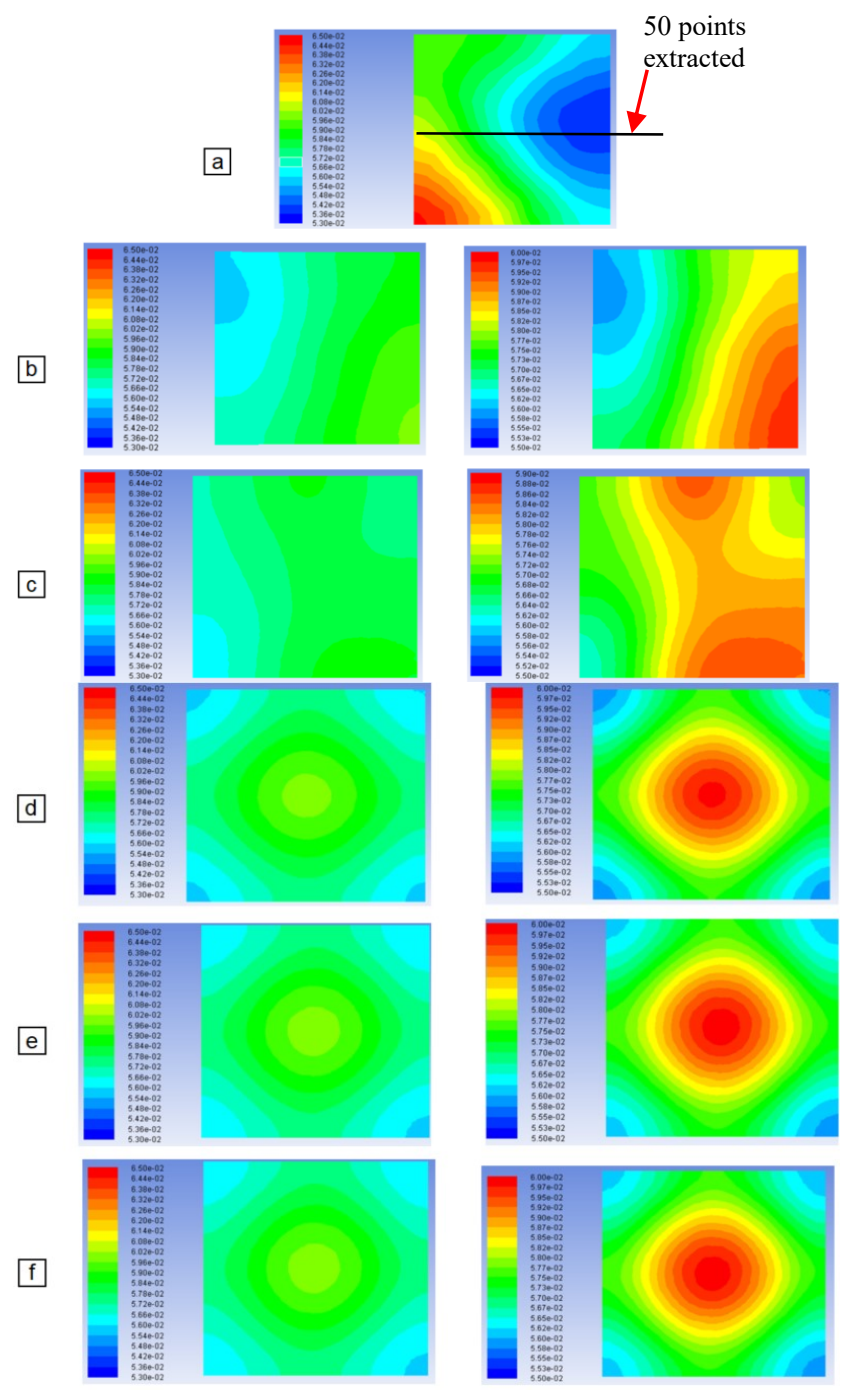

Fig. 8. Velocity fields (u $[\mathrm{m} / \mathrm{s}]$ - velocity magnitude) in transverse plane at distance $10 \mathrm{De}(5 \mathrm{~cm})$ from the metal plate in all the six cases analysed: a) 0.2 , b) 0.62 , c) 1.5 , d) 3 , e) 5.3 , f) 7.3 million elements.

Moreover, figure 8 shows the velocity profiles at $10 \mathrm{De}$ distance from the metal plate for all six cases. It can be observed that at $0.2,0.62$ and 1.5 million elements the profiles resulted are uneven. Beginning with 3 million and even 5.3 million elements no visible variation of the velocity profiles is observed, and these are becoming more and more stable.

So, from a qualitative point of view, we concluded that the 5.3 million elements mesh determines a solution independent of the meshing quality. In order to better assess the mesh quality we analyse the velocity profiles for the six cases with a quantitative approach. As it is indicated in figure 8 -a, we extracted the velocity values in 50 points on the $Z[\mathrm{~mm}]$ axis for all the six mesh levels. The velocity profiles obtained are presented in figure 9 and we can notice that from 3 million elements and above the results are similar.

In the present study, the $10 \mathrm{De}$ velocity profiles are the most relevant to the numerical model because the results at $10 \mathrm{De}$ will constitute the inlet boundary conditions for the solar collector numerical model which will be analysed in the further studies. Moreover, from the experimental studies conducted we observed that at $5 \mathrm{~cm}$ distance from the absorbent plate (10De) the velocity fields become uniform.

Figure 10 presents the temperature fields in al transverse planes discussed were we can observe that there are no visible differences in the temperature fields (phenomena observed in all the 6 cases).

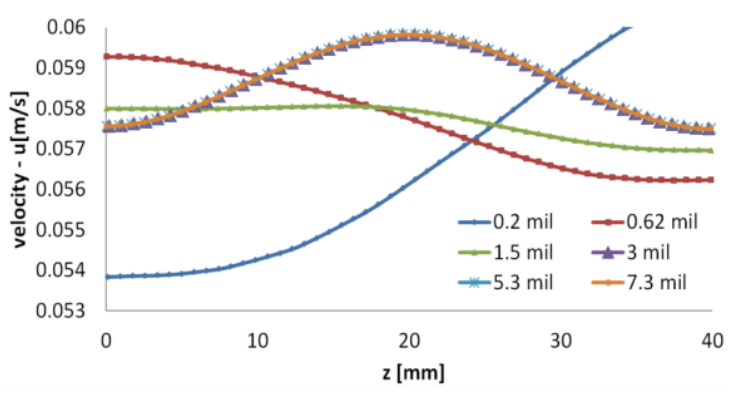

Fig. 9. Velocity profiles (u $[\mathrm{m} / \mathrm{s}]$ - velocity magnitude) quantitative approach.

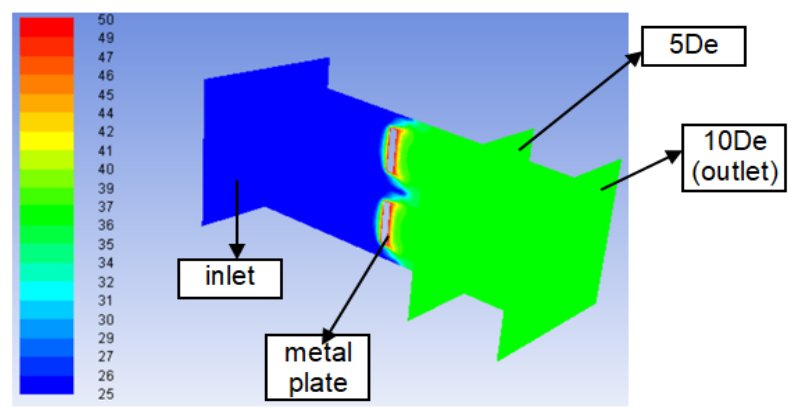

Fig. 10. Temperature fields in transverse planes.

Taking into consideration the above mentioned arguments, by realising a qualitative and quantitative analysis, we can conclude that the 5.3 million cells spatial meshing determines a solution independent of the meshing quality which can be used for the further studies in order to analyse the impact of the orifices geometry on the transpired solar collector.

\section{Conclusions}

In order to perform the mesh independency study, we analysed for all the 6 cases the velocity and temperature profiles in XY longitudinal planes, respectively at 5De and $10 \mathrm{D}$ distance from the metal plate in transverse planes.

If there were no significant differences in the first two situations, we can conclude that from the 5.3 million elements mesh there are no visible variations between the velocity profiles.

Regarding the 10De transverse plane, although small differences appear in the figure 8 , the velocity distribution is relatively uniform with very small variations between 0.055 and $0.06 \mathrm{~m} / \mathrm{s}$. Also, we observed that in the transverse plane there are no visible differences in the temperature fields in all the 6 cases. Considering the imposed boundary conditions for this case, the air temperature at $5 \mathrm{De}$, respectively at $10 \mathrm{De}$ is the same around $37^{\circ} \mathrm{C}$. 
To conclude, following the mesh independency study conducted, we chose the geometry of 5.3 million cells because it is an independent solution to the computing grid and this will be used in the numerical model analysed.

The mesh independency study must be followed by the experimental validation of the numerical model in order to achieve the final numerical model. This analysis will be a subject of further studies.

The numerical model achieved will be used in order to study the airflow through the lobed orifices, to optimize the solar collector geometry, to analyse the impact of orifice geometry on the collectors' efficiency and to realize parametrical studies.

This work was supported by a grant of the Romanian National Authority for Scientific Research, CNCS - UEFISCDI, project number PN-III-P2-2.1-PED-2016-1154.

\section{References}

1. C.V. Croitoru, I. Nastase, F.I. Bode, and A. Meslem, Solar Energy, 131 21-29 (2016).

2. C. Dymond and C. Kutscher, Solar Energy, 60 (5), 291-300 (1997).

3. C. Reichl, K. Kramer, C. Thoma, P. Benovsky, and T. Lemée, Solar Energy, 120 450-463 (2015).

4. B. Molineaux, B. Lachal, and O. Guisan, Solar Energy, 53 (1), 27-32 (1994).

5. L. Gao, H. Bai, and S. Mao, Energy Conversion and Management, 77 690-699 (2014).

6. G.W.E. Van Decker, K.G.T. Hollands, and A.P. Brunger, Solar Energy, 71 (1), 33-45 (2001).

7. E. Osterman, V. Butala, and U. Stritih, Energy and Buildings, 106 125-133 (2015).

8. S. Li, Energy Procedia, (2012).

9. M. Gholampour and M. Ameri, Appl. Energy 164, (2016).

10. M. Badache, D. Rousse, S. Halle, G. Quesada, and Y. Dutil, Energy Procedia, (2012).

11. Amina Meslem, Ilinca Nastase, Florin Bode, and Claudine Beghein, The International Journal of Ventilation, 11 (3), 16 (2012).

12. Amina Meslem, Florin Bode, Ilinca Nastase, and Olivier Martin, Modern Applied Science, 6 (12), 15 (2012).

13. Florin Bode, Ilinca Nastase, and Cristiana Croitoru, Mathematical Modelling in Civil Engineering - Scientific Journal, 7 (4), 7 (2011).

14. Florin Bode, Kodjovi Sodjavi, Amina Meslem, and I. Nastase, SCIENTIFIC BULLETIN University Politehnica of Bucharest, Series D: Mechanical Engineering, 76 (12), 8 (2014).

15. C. Croitoru, I. Nastase, I. Voicu, A. Meslem, and M. Sandu, Energy Procedia, 85 149-155 (2016).
16. ANSYS, Inc "Fluent 19.0 User Guide".

17. Manual:. ANSYS Fluent Chapter 12.4.2 RNG kepsilon Model. accessed march 2018; Available from:

www.sharcnet.ca/Software/Fluent6/html/ug/nod e479.htm. 